\title{
EDITORIAL
}

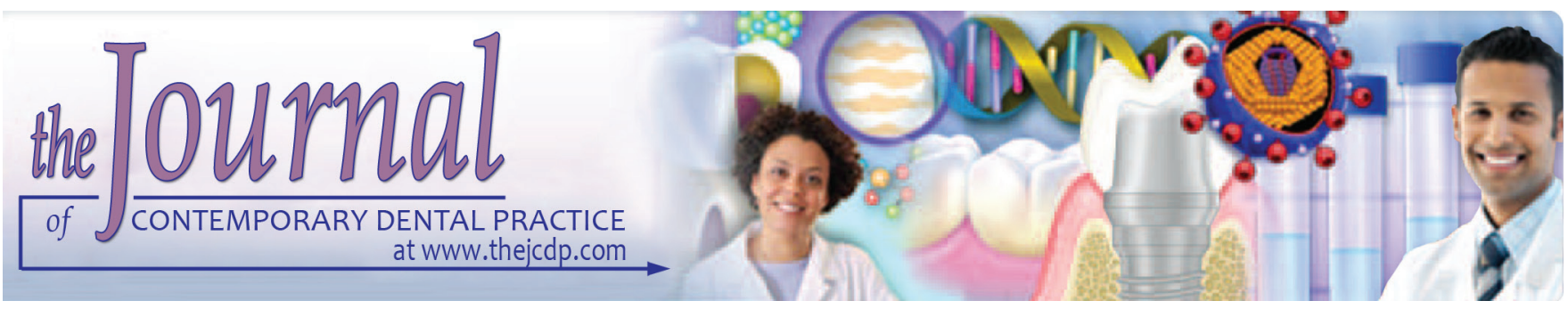

\section{Is the Pen really Mightier than the Scalpel?}

\author{
${ }^{1}$ Gargi S Sarode, ${ }^{2}$ Nilookumari Choudhary, ${ }^{3}$ Sachin C Sarode, ${ }^{4}$ Shankargouda Patil
}

How to cite this article: Sarode GS, Choudhary N, Sarode SC, Patil S. Is the Pen really Mightier than the Scalpel? J Contemp Dent Pract 2017;18(11):987-988.

Source of support: Nil

Conflict of interest: None

\section{INTRODUCTION}

One of the most crucial aspects of cancer diagnosis and treatment is to determine the thin boundary between lesional and normal tissues. So it is important to assess an accurate negative margin and to completely excise the tumor for a prolonged disease-free state and increase the overall survival of the patient.

The conventional method for margin assessment is histopathological analysis, which is labor-intensive, timeintensive, and requires expertise. Another related method is the frozen section preparation which requires skilled technicians and pathologists. Though the technique is quite reliable, it has few disadvantages like freezing artifacts affecting the tissue structure and cell morphology. The intraoperative margin analysis increases the operating time and there is an increase in the risks related to extended anesthesia. Postoperatively, when the margins are found to be positive, the patient has to undergo additional surgery for excision of involved margin, which increases the costs and surgical complications including stress and anxiety. ${ }^{1}$

\footnotetext{
${ }^{1-3}$ Department of Oral Pathology and Microbiology, Dr. D. Y. Patil Dental College and Hospital, Dr. D. Y. Patil Vidyapeeth, Pune Maharashtra, India

${ }^{4}$ Department of Diagnostic Sciences, Division of Oral Pathology College of Dentistry, Jazan University, Jazan, Kingdom of Saudi Arabia

Corresponding Author: Gargi S Sarode, Department of Oral Pathology and Microbiology, Dr. D. Y. Patil Dental College and Hospital, Dr. D. Y. Patil Vidyapeeth, Pune, Maharashtra, India Phone: +919823871462, e-mail: gargi14@gmail.com
}

There are few other methods by which surgical margin assessment can be done. Rapid evaporative ionization mass spectrometry (MS) called iKnife is one of them. It includes an electrocauterization device and MS. It allows to rapidly analyze the vital as well as processed tissues, which helps in real-time margin analysis. ${ }^{2}$ Another important advantage is that this method helps to localize lesional tissue including metastases during resection. ${ }^{2}$ But the technology relies on tissue damage to produce molecular ions. ${ }^{2-4}$ Along with this, the major concern is that the electrosurgical smoke contains irritants, carcinogens, and neurotoxic compounds. Exposure to these compounds may cause health hazards. ${ }^{5}$

Another method is desorption electrospray ionization MS imaging, which is useful for ex vivo cancer diagnosis and to evaluate surgical margin of tissue sections and tissue smears. ${ }^{3}$ The chemical information is obtained directly from the tissue samples. It has a greater advantage of being used for clinical or field application, as it is portable. ${ }^{6}$ The major drawback of this technique is that it cannot be used for in vivo analysis as it includes use of organic solvent sprays, high-pressure nebulizing gas, and high voltages. ${ }^{3,7,8}$ It is also known to have lower spatial resolution (180-200 $\mu \mathrm{m}){ }^{6}$ The MS coupled with ultraviolet lasers and infrared lasers is a laser desorption ionization (LDI)-MS analysis. Since it is reported that the ablation of thin tissue sections with lasers produces a characteristic phospholipid ion population, the laser surgery combined with MS detection can potentially be used during surgical interventions for the real-time, in situ identification of tissues. At the histological level, bulk and vital tissues can be analyzed with real-time identification. ${ }^{9}$ But the disadvantages are as follows: as no spectra are detected at 532, 785, or $1064 \mathrm{~nm}$, the LDI depends on the wavelength of the laser. It causes tissue damage to produce molecular ions for in vivo diagnosis. ${ }^{9,10}$ The method is likewise operationally compelled to a particular surgical methodology. There is a local rise in temperature and the structural proteins are precipitated 
as the laser energy is absorbed by the tissue, which eventually causes boiling of the water content of tissue. Blood alters the resulting spectra as it has its own characteristic phospholipid profile. ${ }^{9}$ The source, sample, and the MS inlet should be aligned and there should be geometrical optimization to achieve a good ion transmission for the other MS methods.

One recent interesting invention we came across was the MasSpec pen. It is a handheld device that is automated, is biocompatible, and has a disposable tip which can be used for nondestructive direct real-time diagnosis of ex vivo and in vivo cancer. Efficient extraction of biomolecules is done with a controlled and automated delivery of a discrete water droplet to the tissue surface. The mass spectra comprises rich molecular profile characterized by a variety of potential cancer biomarkers recognized as metabolites, lipids, and proteins. It has been reported that the database allows prediction of malignancy with a high sensitivity $(96.4 \%)$, specificity $(96.2 \%)$, and a good overall precision $(96.3 \%) .{ }^{3}$ All the components are incorporated within the tip so the geometrical optimization and alignment is not required.

The pen has three primary components: syringe pump, polytetrafluoroethylene tubing conduits, and a sampling probe. The main component of the probe is the polydimethylsiloxane tip that is three-dimensionally printed and disposable. It takes around 10 seconds to perform the entire process that is from sampling of tissue to the acquisition of mass spectra ${ }^{3}$ (Flow Chart 1 ).

MasSpec pen has been tested on human normal tissues and cancerous tissues from ovary, breast, lung, and thyroid. It can obtain nondestructive rich molecular information from tissue samples. It is capable of identifying histologically distinct regions in a single human tissue sample. ${ }^{3}$ These results show that the MasSpec pen can be

Flow Chart 1: Operational steps of the MasSpec pen

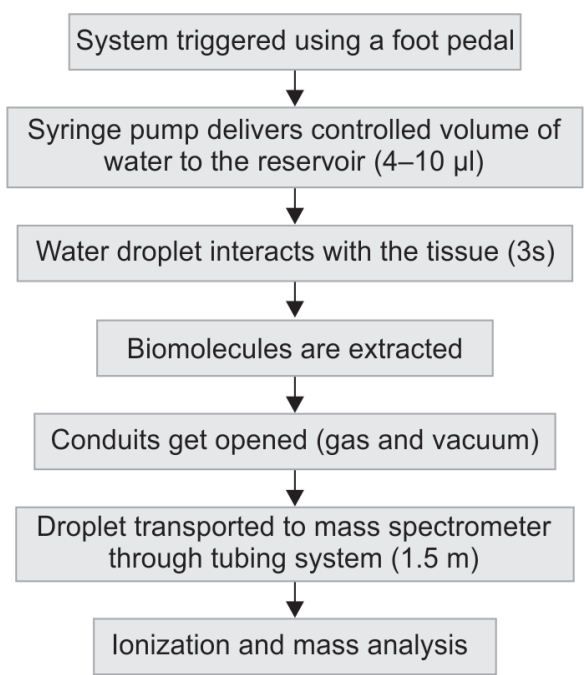

used to detect cancer in margins with mixed composition of normal and cancer cells by the molecular information obtained.

Thus, this recent technology in the diagnosis of cancer can be exploited for oral squamous cell carcinoma (OSCC) detection, diagnosis, and assessment. It can be used intraoperatively also to assure the complete excision of cancerous tissue which will offer a prolonged disease-free state and increase the overall survival. Thus, the MasSpec pen, considering its performance, simple design, and usage with clinically desirable features, enables its use in clinics or primary health care centers for routine screening and diagnosis of OSCC leading to improved patient care and treatment.

\section{REFERENCES}

1. Macario A. What does one minute of operating room time cost? J Clin Anesth 2010 Jun;22(4):233-236.

2. Schäfer KC, Dénes J,Albrecht K, Szaniszló T, Balog J,Skoumal R, Katona M, Tóth M, Balogh L, Takáts Z. In vivo, in situ tissue analysis using rapid evaporative ionization mass spectrometry. Angew Chem Int Ed Engl 2009;48(44):8240-8242.

3. Zhang J, Rector J, Lin JQ, Young JH, Sans M, Katta N, Giese N, Yu W, Nagi C, Suliburk J, et al. Nondestructive tissue analysis for ex vivo and in vivo cancer diagnosis using a handheld mass spectrometry system. Sci Transl Med 2017 Sep;9(406):3968.

4. Balog J, Sasi-Szabóppp L, Kinross J, Lewis MR, Muirhead LJ, Veselkov K, Mirnezami R, Dezs B, Damjanovich L, Darzi A, et al. Intraoperative tissue identification using rapid evaporative ionization mass spectrometry. Sci Transl Med 2013 Jul;5(194):194ra93.

5. Al Sahaf OS, Vega-Carrascal I, Cunningham FO, McGrath JP, Bloomfield FJ. Chemical composition of smoke produced by high-frequency electrosurgery. Ir J Med Sci 2007 Sep;176(3): 229-232.

6. Gemperline E, Chen B, Li L. Challenges and recent advances in mass spectrometric imaging of neurotransmitters. Bioanalysis $2014 \mathrm{Feb} ; 6(4): 525-540$.

7. Eberlin LS, Norton I, Orringer D, Dunn IF, Liu X, Ide JL, Jarmusch AK, Ligon KL, Jolesz FA, Golby AJ, et al. Ambient mass spectrometry for the intraoperative molecular diagnosis of human brain tumors. Proc Natl Acad Sci U S A 2013 Jan;110(5):1611-1616.

8. Jarmusch K, Pirro V, Baird Z, Hattab EM, Cohen-Gadol AA, Cooks RG. Lipid and metabolite profiles of human brain tumors by desorption electrospray ionization-MS. Proc Natl Acad Sci U S A 2016 Feb;113(6):1486-1491.

9. Schäfer KC, Szaniszló T, Günther S, Balog J, Dénes J, Keserű M, Dezso B, Tóth M, Spengler B, Takáts Z. In situ, real-time identification of biological tissues by ultraviolet and infrared laser desorption ionization mass spectrometry. Anal Chem 2011 Mar;83(5):1632-1640.

10. Fatou B, Saudemont P, Leblanc E, Vinatier D, Mesdag V, Wisztorski M, Focsa C, Salzet M, Ziskind M, Fournier I. In vivo real-time mass spectrometry for guided surgery application. Sci Rep 2016 May;6:25919. 